\title{
Three-body approach to proton-hydrogen charge exchange and elastic scattering
}

\author{
E. O. Alt \\ Institut für Physik, Universität Mainz, D-55099 Mainz, Germany \\ A. S. Kadyrov \\ Electronic Structure of Materials Centre, The Flinders University of South Australia, GPO Box 2100, Adelaide 5001, Australia \\ A. M. Mukhamedzhanov \\ Cyclotron Institute, Texas A\&M University, College Station, Texas 77843
}

(Received 2 October 1998)

\begin{abstract}
The impact-parameter Faddeev approach to atomic three-body collisions which has been developed for, and successfully applied to, ion-atom scattering processes, has now been developed further by including, instead of the Coulomb potentials, the full two-particle off-shell Coulomb $T$ matrices in all "triangle" contributions to the effective potentials. Results of calculations of proton-hydrogen collisions with only the ground states of the hydrogen retained in both the direct and the rearrangement channels are presented. Total and differential electron transfer, as well as differential elastic scattering cross sections, are obtained simultaneously in very good agreement with experiment, over a wide range of (nonrelativistic) incident energies.
\end{abstract}

[S1050-2947(99)01407-9]

PACS number(s): $34.80 . \mathrm{Bm}$

\section{INTRODUCTION}

Seven decades have passed since Oppenheimer [1] and Brinkmann and Kramers [2] (OBK) calculated for the first time the electron transfer in proton-hydrogen collisions. The lowest-order approximation that was used neglected the proton-proton interaction, resulting in an overestimation of the total cross section almost by an order of magnitude. More than two decades later, Jackson and Schiff (JS) [3] showed that inclusion of the internuclear interaction could bring down the cross section close to experiment. Soon after, it was realized that also the JS approximation faces inherent difficulties, e.g., when applied to the calculation of differential cross sections. Nevertheless, these investigations did lay the basis for the considerable progress, achieved over the years, in the theoretical understanding of the process of interest.

However, in our opinion even this simplest chargetransfer reaction is still lacking a satisfactory theoretical description in the moderate- to high-energy region. As far as the total cross section at higher energy is concerned, two DWBA-type methods should be mentioned as being superior to all other theories, namely the continuum distorted wave (CDW) $[4,5]$ and the boundary-corrected first Born (B1B) [5-7] approximations. But a convincing test of the quality of theoretical models must include reproduction of the experimental data also for the differential cross sections. Here, it turns out that both CDW and B1B approximations are considerably less successful. The deeper reason for this shortcoming is that both are essentially one-channel approximations; that is, contributions to the rearrangement channel coming either from other reaction channels or from the interference between different states in a given channel are not included. This entails that not sufficient allowance is made for the constraints provided by two-body, and in particular not by three-body, unitarity.

In view of these facts, a method is still called for which would properly take into account all possible reaction channels, and correctly reproduce the total cross section as a consequence of the successful description of the corresponding differential cross sections.

We believe that for such an undertaking, the "three-body Faddeev approach," although it has not been applied to atomic collision problems as widely as other traditional methods, represents the appropriate framework. To be sure, its application to atomic collisions becomes tedious mainly, as stated in the recent review on energetic ion-atom collision theories [8], because of the complicated singularity structure of the two-particle off-shell Coulomb $T$ matrix which is the basic dynamical ingredient in that formalism. For, as is well known, the Coulomb $T$ matrix does not have a well-defined on-shell limit and, in the case of attraction, possesses an infinite number of bound-state poles. In spite of these problems, first calculations were published in the early 1970s (see [9] for a review). But soon this approach ceased attracting interest, partly because of the aforementioned difficulties, but partly also because a number of - as we think-incorrect calculations (e.g., [10-13], to be discussed later on) led to unsatisfactory results.

Such a discouraging history notwithstanding, the threebody theory can, in our opinion, be utilized with advantage for the investigation of atomic reactions. This belief is based not only on our previous calculations of electron transfer in ion-atom collisions using the few-body integral equations formalism [14-17], but also on recent Faddeev calculations by other authors (see [18] and references therein), and on our recent investigations of reaction mechanisms containing the two-particle off-shell Coulomb $T$ matrix [19-22].

Some time ago the impact parameter Faddeev approach 
(IPFA) to ion-atom collisions was developed [14] and applied to the calculation of different electron-transfer reactions $[15,16]$. It was based on the effective-two-body formulation of the three-body theory as proposed by Alt, Grassberger, and Sandhas (AGS) [23], appropriately modified to accommodate long-range Coulomb interactions $[24,25]$. These coupled integral equations connecting the amplitudes for all binary processes were then written in on-shell approximation, i.e., the exact intermediate-state twofragment propagator was approximated by the corresponding energy conserving $\delta$ function. Instead of making a partial wave expansion to further reduce the dimensionality of the integral equations, transformation to the impact parameter representation was used. This led to a coupled system of algebraic equations for the two-cluster amplitudes satisfying - at least partially - the constraints from two- and three-particle unitarity. In the concrete applications, the effective potentials occurring therein were taken into account only in the lowest-order approximation corresponding to the electron-transfer ("pole") mechanism. Calculations of total and partial electron-transfer cross sections showed on the whole good agreement with available experimental data, over a wide range of reactions and energies. But at high energies this approach overestimated the data, giving only qualitative results, due to the neglect of higher-order terms in effective potentials.

To simplify the treatment of the next-order ("triangle") terms in the effective potentials at higher energies, we later developed the so-called three-body eikonal approach (TBEA) [17]. There again, AGS effective-two-body equations were formulated for the appropriate amplitudes for scattering and charge exchange. In addition to the "pole" terms, explicit expressions for the "triangle" contributions to the effective potentials were derived, but only after the two-body Coulomb $T$ operators occurring therein had been taken in Born approximation, i.e., after they had been replaced by the corresponding Coulomb potentials. This allowed us to avoid the above-mentioned singularities of the $T$ matrix. Though our calculations showed that for reactions with nonvanishing Coulomb interaction in the ingoing and/or outgoing channel the TBEA leads to considerable improvement in the description of total and partial electron transfer cross sections, it nevertheless was still not capable of describing the data on differential cross sections.

Because of this shortcoming it was concluded that use of the exact Coulomb $T$ matrix cannot be bypassed. As a first step towards this goal, extensive investigations of the various exact "triangle" amplitudes, which occur in both the exchange and the direct scattering channels, have been performed recently [19-21]. For the case of an attractive interaction, a "new" representation of the Coulomb $T$ matrix has been derived which turned out to be very efficient for numerical purposes [21].

The objective of the present paper is to demonstrate that the further development of the IPFA, brought about by exactly including the two-particle off-shell Coulomb $T$ matrices in the first-order terms of the effective potentials, does indeed lead to a very satisfactory description, not only of total exchange cross sections but also of differential cross sections for direct and exchange scattering.

The plan of the paper is as follows. The IPFA is briefly outlined in Sec. II. The results of calculations are presented in Sec. III. Finally, Sec. IV contains our conclusions.

\section{FORMALISM}

We consider a system of two protons and one electron. In order to describe direct scattering and electron transfer in collisions of protons with hydrogen atoms, we shall use the effective-two-body formulation of the AGS three-body equations [23]. In this section we outline the basic idea of the IPFA for the present, simplified case.

Assume a decomposition of the two-body $T$-operator $T_{\alpha}$ into two terms,

$$
T_{\alpha}(\hat{z})=T_{\alpha}^{\prime}(\hat{z})+T_{\alpha}^{\mathrm{sep}}(\hat{z}),
$$

where

$$
T_{\alpha}^{\mathrm{sep}}(\hat{z})=\sum_{m=1}^{N_{\alpha}}\left|\chi_{\alpha m}(\hat{z})\right\rangle g_{\alpha m}(\hat{z})\left\langle\chi_{\alpha m}\left(\hat{z}^{*}\right)\right|
$$

is chosen as a sum of separable terms representing $N_{\alpha}$ bound states of the particle pair $(\beta, \gamma)_{m}$ with quantum numbers " $m$ " and binding energies $\hat{E}_{\alpha m}$, and $T_{\alpha}^{\prime}(\hat{z})$ is the (possibly nonseparable) remainder. Here,

$$
g_{\alpha m}(\hat{z})=\left(\hat{z}-\hat{E}_{\alpha m}\right)^{-1} .
$$

Consider the reaction initiating from a channel where particle $\alpha$ is free, and particles $\beta$ and $\gamma$ are bound with wave function $\left|\psi_{\alpha m}\right\rangle$ belonging to the energy $\hat{E}_{\alpha m} ; \mathbf{q}_{\alpha}$ denotes the channel relative momentum. Similarly, let the final state be characterized by the relative momentum $\mathbf{q}_{\beta}^{\prime}$ between particle $\beta$ and the bound state (with energy $\hat{E}_{\beta n}$ ) of the other two particles. Then the corresponding reaction amplitude $\mathcal{T}_{\beta n, \alpha m}\left(\mathbf{q}_{\beta}^{\prime}, \mathbf{q}_{\alpha} ; z\right)$ can be found by solving the following set of coupled Lippmann-Schwinger-type equations:

$$
\begin{aligned}
\mathcal{T}_{\beta n, \alpha m} & \left(\mathbf{q}_{\beta}^{\prime}, \mathbf{q}_{\alpha} ; z\right) \\
= & \mathcal{V}_{\beta n, \alpha m}\left(\mathbf{q}_{\beta}^{\prime}, \mathbf{q}_{\alpha} ; z\right) \\
& +\sum_{\nu, r} \int d \mathbf{q}_{\nu}^{\prime \prime} \mathcal{V}_{\beta n, \nu r}\left(\mathbf{q}_{\beta}^{\prime}, \mathbf{q}_{\nu}^{\prime \prime} ; z\right) \mathcal{G}_{0 ; \nu r}\left(\mathbf{q}_{\nu}^{\prime \prime} ; z\right) \\
& \times \mathcal{T}_{\nu r, \alpha m}\left(\mathbf{q}_{\nu}^{\prime \prime}, \mathbf{q}_{\alpha} ; z\right) .
\end{aligned}
$$

In fact, provided the so-called form factors $\left|\chi_{\alpha m}(z)\right\rangle$ are chosen such that on the energy shell, i.e., for $E=q_{\alpha}^{2} / 2 M_{\alpha}$ $+\hat{E}_{\alpha m}=q_{\beta}^{\prime 2} / 2 M_{\beta}+\hat{E}_{\beta n}$,

$$
G_{0}(z)\left|\chi_{\alpha m}(z)\right\rangle\left|\mathbf{q}_{\alpha}\right\rangle^{z \rightarrow E+i 0} \rightarrow\left|\psi_{\alpha m}\right\rangle\left|\mathbf{q}_{\alpha}\right\rangle,
$$

and analogously for $\left|\chi_{\beta n}(z)\right\rangle$, the on-shell value of $\mathcal{T}_{\beta n, \alpha m}\left(\mathbf{q}_{\beta}^{\prime}, \mathbf{q}_{\alpha} ; E+i 0\right) \equiv \mathcal{T}_{\beta n, \alpha m}\left(\mathbf{q}_{\beta}^{\prime}, \mathbf{q}_{\alpha}\right)$ is the physical reaction amplitude we are looking for. Here, $G_{0}(z)$ $=\left(z-H_{0}\right)^{-1}$ is the free resolvent and $M_{\alpha}=m_{\alpha}\left(m_{\beta}\right.$ $\left.+m_{\gamma}\right) /\left(m_{\alpha}+m_{\beta}+m_{\gamma}\right)$ is the reduced mass of the two fragments in channel $\alpha$. The effective free Green function is defined as 

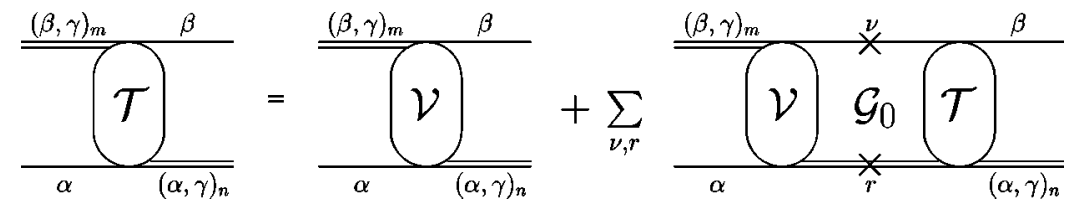

FIG. 1. Graphical representation of the integral equation (10). The crosses indicate that the particles propagating in the intermediate state are put on the energy shell by taking into account only the $\delta$-function part of the effective free propagator.

$$
\mathcal{G}_{0 ; \alpha m}\left(\mathbf{q}_{\alpha} ; z\right)=g_{\alpha m}\left(z-q_{\alpha}^{2} / 2 M_{\alpha}\right)
$$

and describes the free relative motion of particle $\alpha$ and the bound pair $(\beta, \gamma)_{m}$. The effective potentials

$$
\begin{aligned}
\mathcal{V}_{\beta n, \alpha m}\left(\mathbf{q}_{\beta}^{\prime}, \mathbf{q}_{\alpha} ; z\right):= & \left\langle\mathbf{q}_{\beta}^{\prime}\right|\left\langle\chi_{\beta n}\left(z^{*}\right)\right| G_{0}(z) U_{\beta \alpha}^{\prime}(z) \\
& \times G_{0}(z)\left|\chi_{\alpha m}(z)\right\rangle\left|\mathbf{q}_{\alpha}\right\rangle
\end{aligned}
$$

are to be calculated by using the following AGS equations for the "reduced" three-body operators $\left(\bar{\delta}_{\beta \alpha}=1-\delta_{\beta \alpha}\right)$ :

$$
U_{\beta \alpha}^{\prime}(z)=\bar{\delta}_{\beta \alpha} G_{0}^{-1}(z)+\sum_{\nu} U_{\beta \nu}^{\prime}(z) G_{0}(z) T_{\nu}^{\prime}(z) \bar{\delta}_{\nu \alpha}
$$

The equations presented so far are exact. However, in order to make their practical solution feasible, we resort to two simplifications. First, we use the on-shell approximation

$$
\begin{gathered}
\mathcal{G}_{0 ; \nu r}\left(\mathbf{q}_{\nu}^{\prime \prime} ; E+i 0\right) \rightarrow-i \pi \delta\left(E-q_{\nu}^{\prime \prime 2} / 2 M_{\nu}-\hat{E}_{\nu r}\right), \\
\nu=\alpha, \beta, \gamma,
\end{gathered}
$$

which is well justified at the high energies considered but limits the applicability of our approach to low energies. In this way we obtain for the on-shell amplitudes $\left(\hat{\mathbf{q}}_{\nu}^{\prime \prime}:=\mathbf{q}_{\nu}^{\prime \prime} / q_{\nu}^{\prime \prime}\right)$

$$
\begin{aligned}
\mathcal{T}_{\beta n, \alpha m}\left(\mathbf{q}_{\beta}^{\prime}, \mathbf{q}_{\alpha}\right)= & \mathcal{V}_{\beta n, \alpha m}\left(\mathbf{q}_{\beta}^{\prime}, \mathbf{q}_{\alpha}\right) \\
& -i \pi \sum_{\nu, r} M_{\nu} q_{\nu} \int d \Omega_{\mathbf{q}_{\nu}^{\prime \prime}} \mathcal{V}_{\beta n, \nu r}\left(\mathbf{q}_{\beta}^{\prime}, q_{\nu} \hat{\mathbf{q}}_{\nu}^{\prime \prime}\right) \\
& \times \mathcal{T}_{\nu r, \alpha m}\left(q_{\nu} \hat{\mathbf{q}}_{\nu}^{\prime \prime}, \mathbf{q}_{\alpha}\right),
\end{aligned}
$$

with the magnitude of the intermediate-state momentum fixed by the energy shell condition (9), i.e., $q_{\nu}$ $\equiv \sqrt{2 M_{\nu}\left(E-\hat{E}_{\nu r}\right)}$. Equation (10) is represented in diagrammatic form in Fig. 1. After transformation of Eq. (10) into the impact parameter representation we end up with a set of coupled algebraic equations [14]

$$
\begin{aligned}
\mathcal{T}_{\beta n, \alpha m}(\rho)= & \mathcal{V}_{\beta n, \alpha m}(\rho) \\
& -\frac{i}{4 \pi} \sum_{\nu, r} M_{\nu} q_{\nu}^{-1} \mathcal{V}_{\beta n, \nu r}(\rho) \mathcal{T}_{\nu r, \alpha m}(\rho)
\end{aligned}
$$

with $\rho$ denoting the impact parameter. The effective potentials in the impact parameter representation, $\mathcal{V}_{\beta n, \alpha m}(\rho)$, are defined as Hankel transforms of corresponding momentum space matrix elements $\mathcal{V}_{\beta n, \alpha m}\left(\mathbf{q}_{\beta}^{\prime}, \mathbf{q}_{\alpha}\right)$.

Equation (8) can, e.g., be solved by iteration yielding the so-called "quasi-Born expansion" of the (on-shell) effective potentials

$$
\begin{aligned}
\mathcal{V}_{\beta n, \alpha m} & \left(\mathbf{q}_{\beta}^{\prime}, \mathbf{q}_{\alpha}\right) \\
= & \bar{\delta}_{\beta \alpha}\left\langle\mathbf{q}_{\beta}^{\prime}\left|\left\langle\psi_{\beta n}\left|E-H_{0}\right| \psi_{\alpha m}\right\rangle\right| \mathbf{q}_{\alpha}\right\rangle \\
& +\sum_{\nu} \bar{\delta}_{\beta \nu} \bar{\delta}_{\nu \alpha}\left\langle\mathbf{q}_{\beta}^{\prime}\right|\left\langle\psi_{\beta n}\right| T_{\nu}^{\prime}(E+i 0) \\
& \times\left|\psi_{\alpha m}\right\rangle\left|\mathbf{q}_{\alpha}\right\rangle+\sum_{\nu, \mu} \bar{\delta}_{\beta \nu} \bar{\delta}_{\nu \mu} \bar{\delta}_{\mu \alpha}\left\langle\mathbf{q}_{\beta}^{\prime}\right|\left\langle\psi_{\beta n}\right| T_{\nu}^{\prime}(E+i 0) \\
& \times G_{0}(E+i 0) T_{\mu}^{\prime}(E+i 0)\left|\psi_{\alpha m}\right\rangle\left|\mathbf{q}_{\alpha}\right\rangle+\cdots
\end{aligned}
$$

In writing down the right-hand side, use has been made of condition (5). The first term corresponds to the so-called "pole" diagram, the second to three different "triangle" graphs, and the third to the double-rescattering contributions (i.e., two consecutive scatterings in different particle pairs). The first two terms are represented graphically in Fig. 2. Now, the second simplification consists in cutting the expansion (12) after the terms of first order in $T_{\nu}^{\prime}$; in other words, double- and higher-order rescattering contributions are neglected. This approximation clearly restricts the applicability to energies well below the region where the Thomas peak starts emerging. We remark that the decomposition (1) has been made for the attractive electron-proton (ep) $T$ matrix only. Consequently, for that subsystem the residual $T$ matrix $T_{\mathrm{ep}}^{\prime}$ is given as in Eq. (1) while for the proton-proton (pp) subsystem we simply have $T_{\mathrm{pp}}^{\prime}=T_{\mathrm{pp}}^{C}$, i.e., $N_{\mathrm{pp}}=0$.

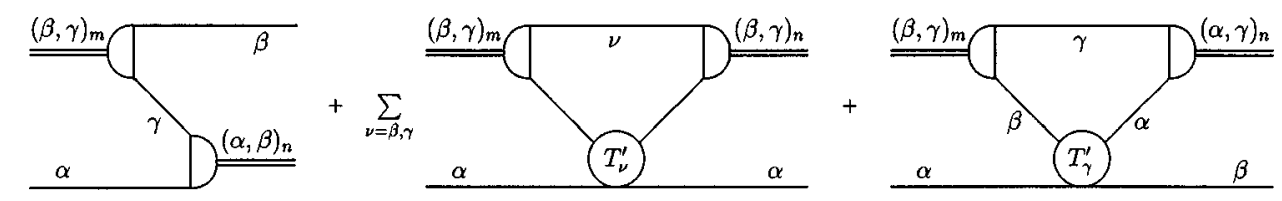

FIG. 2. Graphical representation of the first two terms in the expansion (12) of the effective potentials. Semicircles indicate the form factors satisfying Eq. (5). 
The impact parameter representation of the "pole" contribution for transitions between arbitrary hydrogenic states has already been given in analytical form in [14]. This is not possible for the "triangle" amplitudes so that there a different procedure had to be pursued. Namely, at first the exact first-order terms were calculated numerically in the momentum representation, as described in Refs. [19-21]. Since they turned out to be rather smooth functions of the incident energy and scattering angle, in a second step the Hankel transformation to the impact parameter space could be done numerically without difficulties.

We remark that in order to reliably perform the numerical integrations in those "triangle" amplitudes which contain the attractive Coulomb $T$ matrix, a "new" representation of the latter had to be developed for negative energies such that the infinity of bound state poles are displayed in a numerically convenient form. In fact, the representation derived has the poles given explicitly as zeros of a simple function and otherwise contains smooth integrals along the real axis only [21].

\section{CALCULATIONS AND RESUltS}

In this section we present the results of our calculations of total and differential electron transfer and of differential elastic scattering cross sections in proton collisions with hydrogen atoms in their ground state. In the present work we confine ourselves to the case when only the $1 s$ state of hydrogen is retained explicitly in all possible reaction channels [i.e., $N_{\text {ep }}=1$ in Eq. (2)] and the remainder amplitude $T_{\text {ep }}^{\prime}$ contains only the continuum contribution of $T_{\mathrm{ep}}^{C}$; this will be called the $1 s-1 s$ approximation. Within these model limitations, two-body (elastic and exchange) unitarity is exactly, and three-body unitarity at least partially, satisfied by our amplitudes.

The various transition amplitudes are obtained by solving the set of coupled linear equations in the impact parameter representation (11). The expansion of the effective potential has been truncated after the first-order contribution in Eq. (12). The first ("zeroth-order") term is, in the $1 s-1 s$ case, just the OBK amplitude. The sum of the first two terms pertaining to the exchange channel gives the Chen-Kramer (CK) amplitude [26]. If, in addition, in the latter the off-shell Coulomb $T$ matrix is approximated by the potential, we arrive at the JS amplitude. Hence, restricting ourselves to the first two terms in the expansion (12) means that in our approach the CK amplitude plays the role of the effective potential for the transfer channel while the two diagonal first-order terms play the same role for the direct channel. But we emphasize that the effective potentials are to be inserted into Eqs. (4), or after transformation to the impact parameter space into Eq. (11), to yield the various reaction amplitudes. Diagrammatically this procedure consists in substituting in Fig. 1 for the effective potentials all the diagrams of Fig. 2.

We remark that for the case considered presently (no Coulomb distortions in the initial and final state) the JS is identical to the B1B amplitude. However, we will reserve the notation B1B for cross sections summed over all final states, as is done in $[6,7]$.

Our results are presented in Figs. 3-12, and compared with calculations by other authors and with experimental

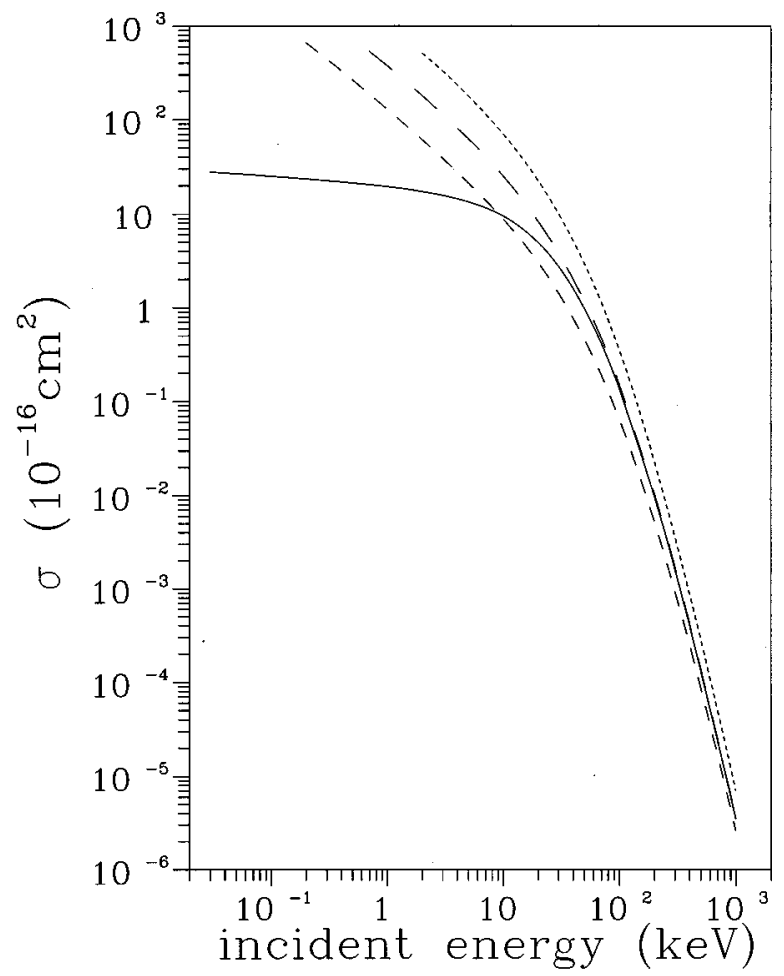

FIG. 3. Integrated cross sections for electron capture by $\mathrm{H}^{+}$ from $\mathrm{H}(1 s)$ : solid line, present results $(1 s)$; dotted line, OBK $(1 s)$ [2]; short-dashed line, JS (1s) [3]; long-dashed line, CK (1s) [26].

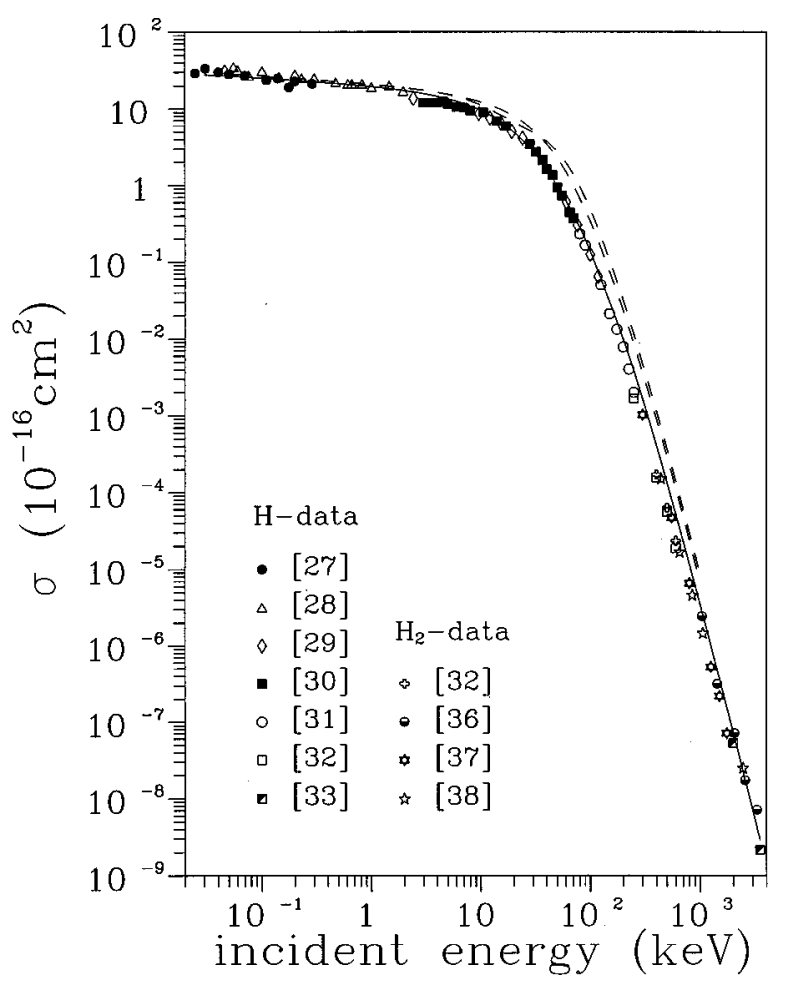

FIG. 4. Same as in Fig. 3 except upper $(\Sigma)$ and lower $(1 s)$ dashed lines (see text), TBEA [17]. Experimental data are from Refs. [27-33,36-38]. Note that the data for the $\mathrm{H}_{2}$ target are transformed for the $\mathrm{H}$ target, according to [34] and [35]. 


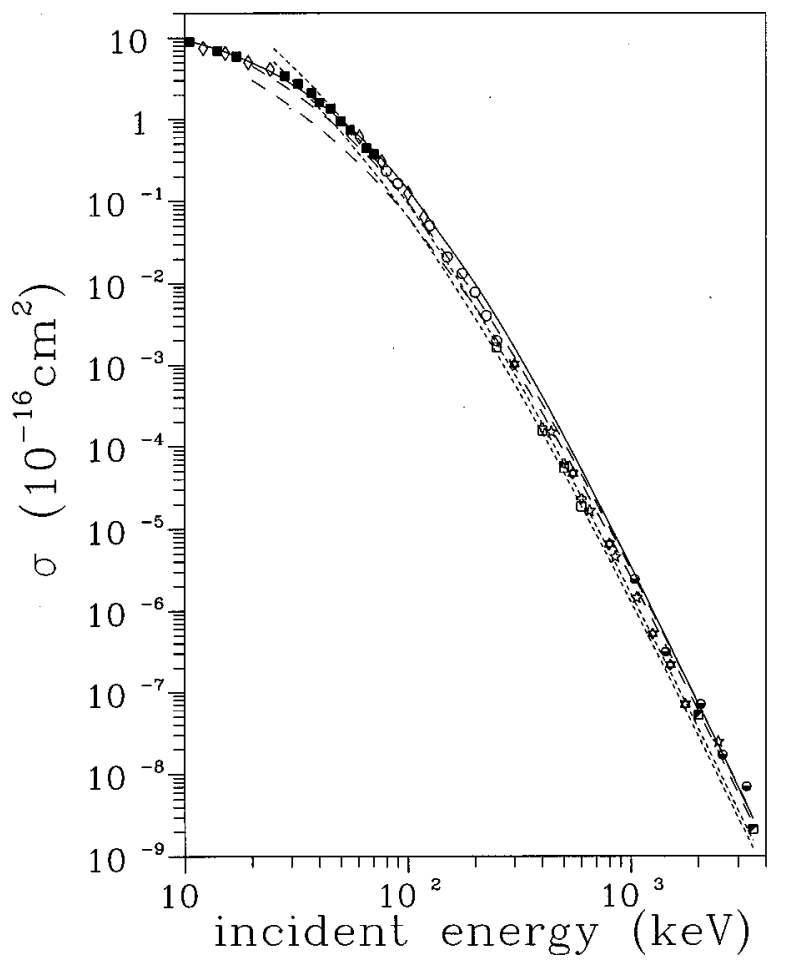

FIG. 5. Same as in Fig. 4 except for the following: dotted lines, CDW [5]; dashed lines, B1B [7]; in each case, the lower line is $1 s$; the upper line is $\Sigma$; see text.

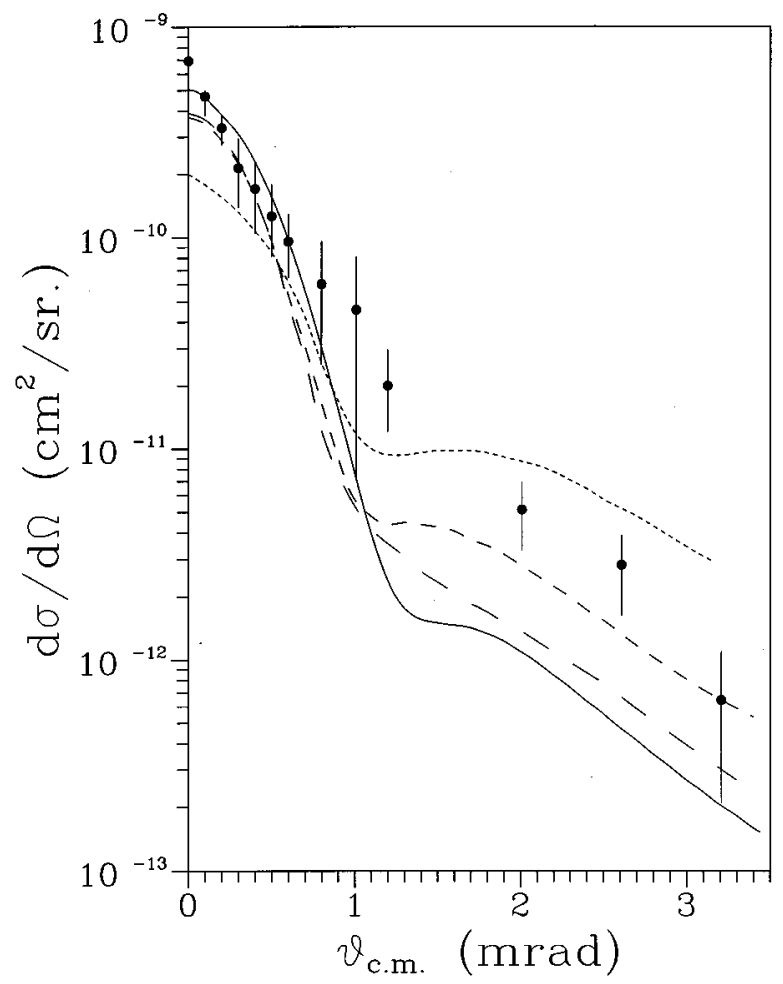

FIG. 6. Differential cross section for electron capture by $\mathrm{H}^{+}$ from $\mathrm{H}(1 s)$ at $25 \mathrm{keV}$ : solid line, present results $(1 s)$; dotted line, CDW (1s) [5]; short-dashed line, B1B $(\Sigma)$ [6]; long-dashed line, MS $(1 s)$ [39]. Experimental points $(\Sigma)$ are from [40].

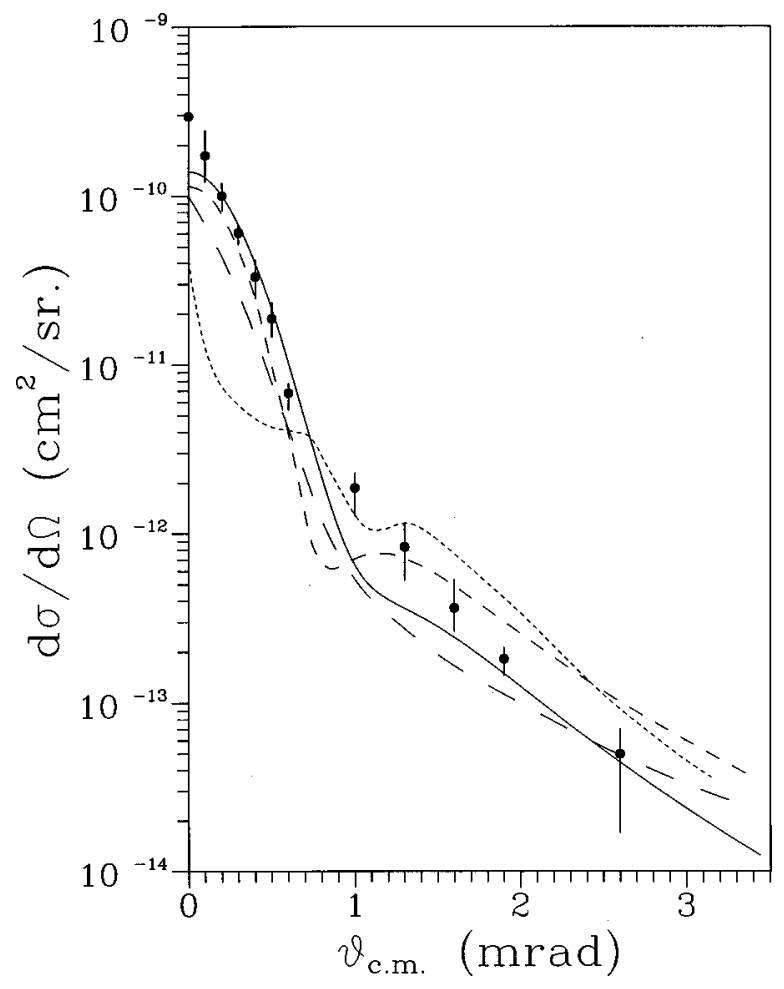

FIG. 7. Same as in Fig. 6 but for $60 \mathrm{keV}$.

data. Total electron-transfer cross sections are displayed in Figs. 3-5. The pairs of curves in Figs. 4 and 5 with identical characterization are always the $1 s-1 s$ total cross section (represented by the curve which lies lower at high energies, also denoted by " $1 s$ "), and the cross section summed over all final states (upper curve, denoted by " $\Sigma$ ",), in the corre-

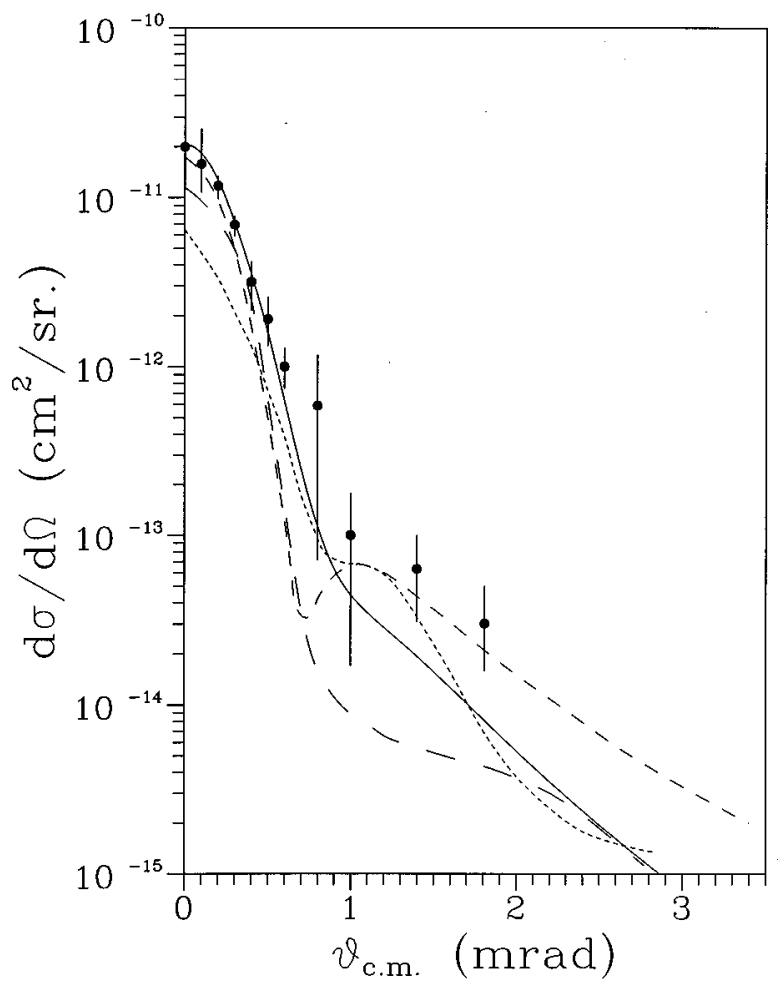

FIG. 8. Same as in Fig. 6 but for $125 \mathrm{keV}$. 


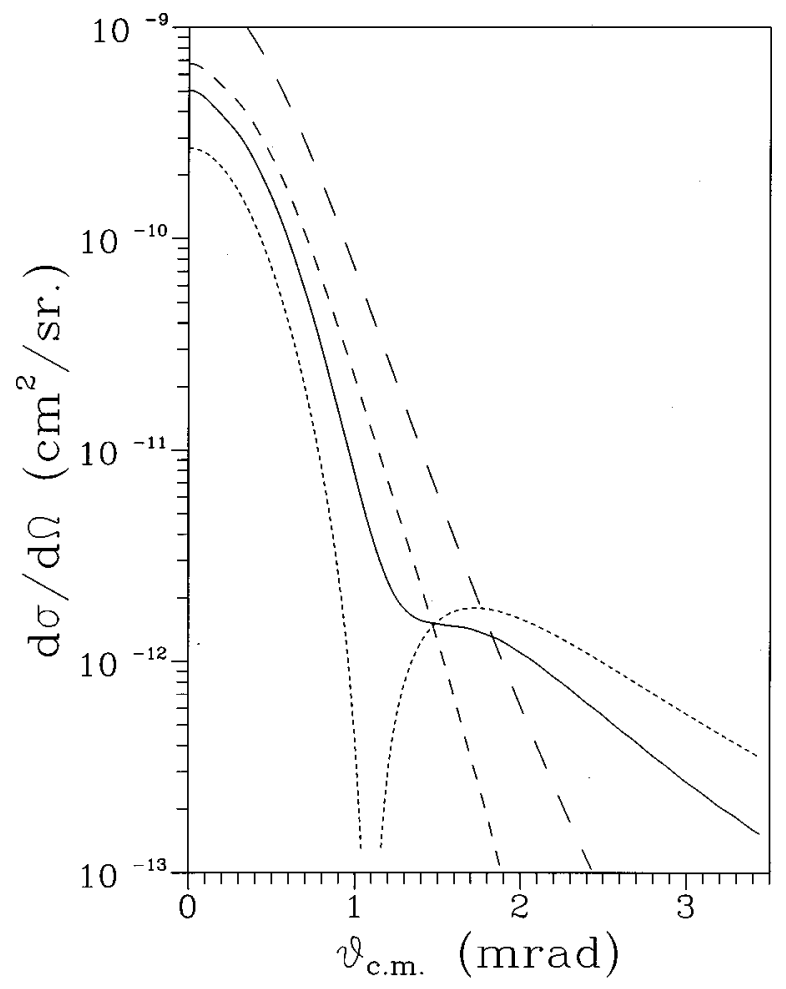

FIG. 9. Same as in Fig. 6 except for the following: long-dashed line, OBK ( $1 s)$ [2]; dotted line, JS (1s) [3]; short-dashed line, CK (1s) [26].

sponding approaches. This provides a hint at the possible size of the correction to our present $1 s-1 s$ cross section which must be expected to result from summation over all final states.

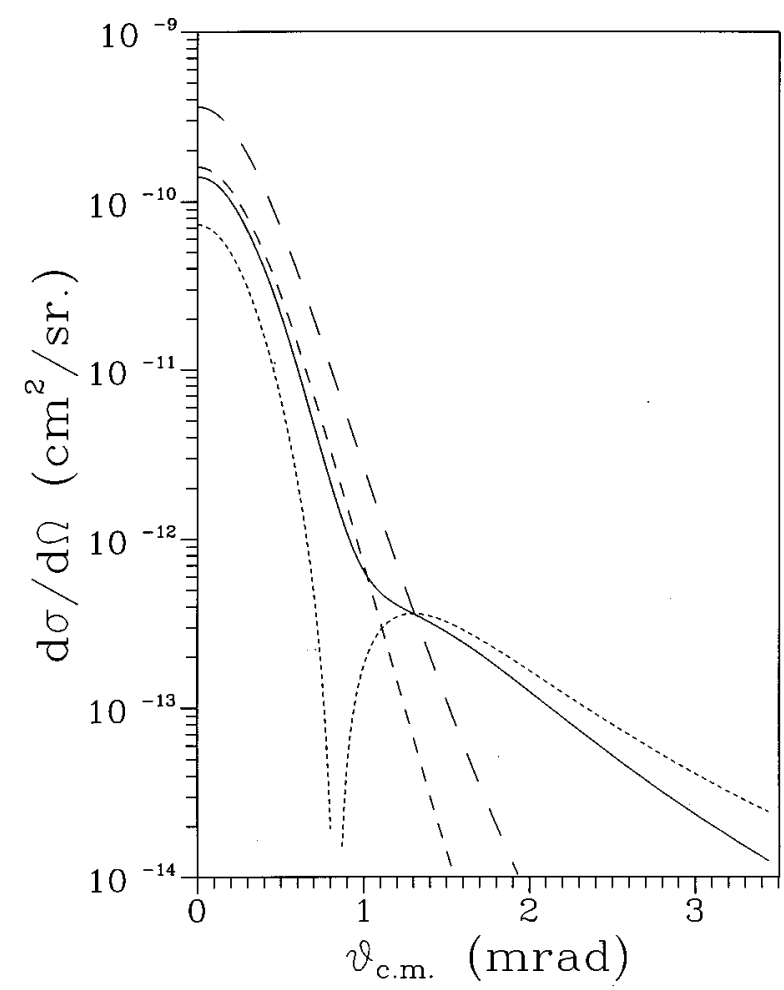

FIG. 10. Same as in Fig. 9 but for $60 \mathrm{keV}$.

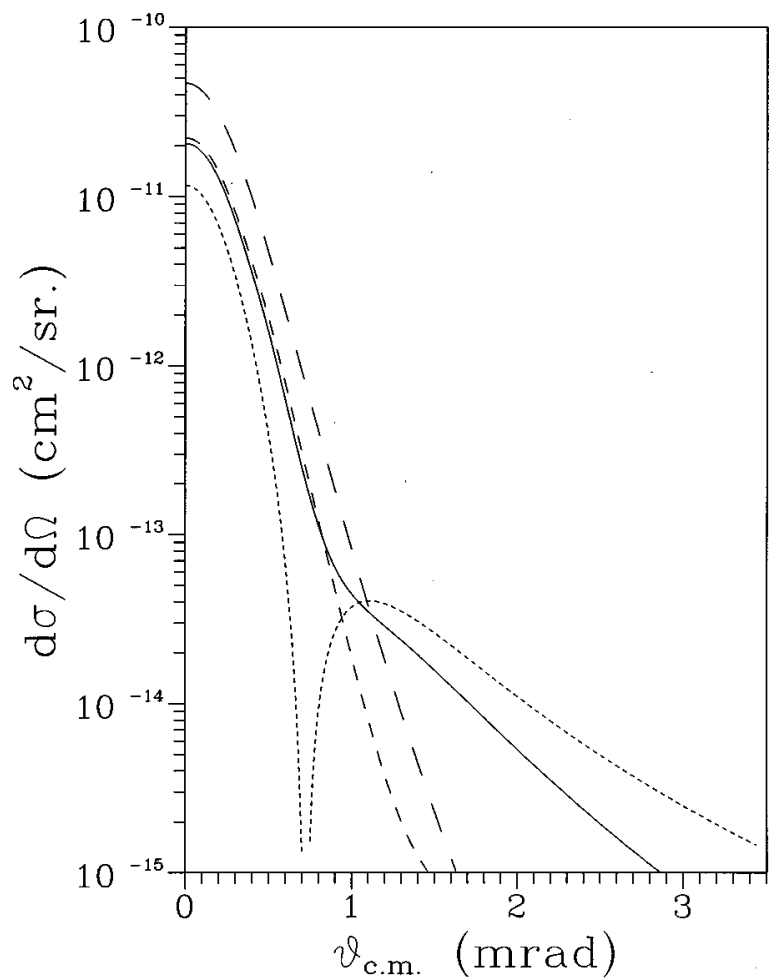

FIG. 11. Same as in Fig. 9 but for $125 \mathrm{keV}$.

As can be inferred from Fig. 3, our values lie between OBK and JS and coincide with CK at higher energies. This latter "coincidence" can easily be understood because the on-shell approximation (11) approaches the Born approximation which for the rearrangement amplitude is identical to the

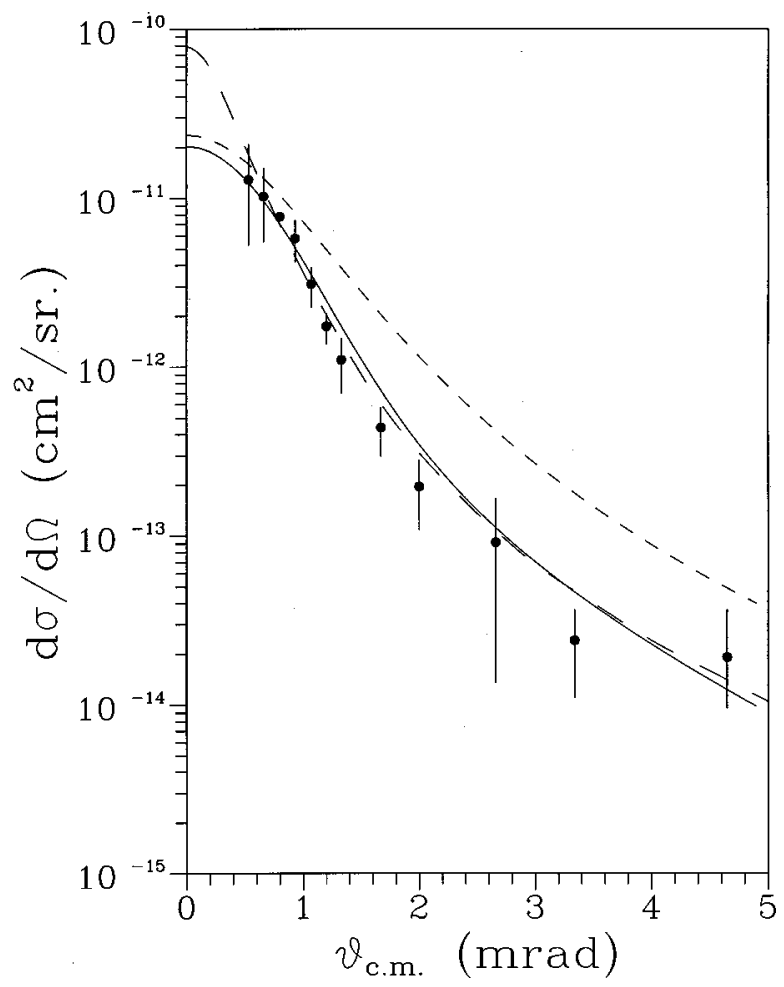

FIG. 12. Differential elastic scattering cross sections of $\mathrm{H}^{+}$on $\mathrm{H}(1 s)$ at $60 \mathrm{keV}$ : solid line, present results; short-dashed line, FBA; long-dashed line, MS [42]. The experimental data are from [44]. 
driving CK term. That the use of the exact "triangle" graphs (i.e., calculated with the full Coulomb $T$ matrix) in the effective potentials is instrumental in reducing the cross section for energies beyond a few $\mathrm{keV}$ is demonstrated in Fig. 4, which shows comparison with the " $1 s$ " (at higher energies the lower-lying dashed curve) TBEA results of [17], where only the approximate "triangle" terms (in the direct scattering channel) had been employed. Finally, in Fig. 5 the highenergy part of our total cross section is compared with that following from the most sophisticated high-energy models, namely CDW and B1B. Inspection reveals that our approach leads to nearly as good agreement as CDW and B1B. But, in contrast to the CDW and B1B (but also to the OBK, JS, and CK) amplitudes, our reaction amplitude is unitary at the twobody (and partially also on the three-body) level. This fact is vital for reducing its value at lower energies, with the result that at the same time we also achieve perfect agreement with the data in the intermediate- and low-energy region, as shown in Fig. 4. Summarizing, both fulfillment of the unitarity constraints and use of the exact triangle amplitudes taken together eventually lead to a very good agreement with experiment, over a wide range of energy.

Here we would like to comment on the Faddeev calculations of Sil and co-workers. If, in our approach, we evaluate the "triangle" diagrams in both the direct and the transfer channels in Born approximation only, i.e., with $T_{\alpha}^{C}$ replaced by $V_{\alpha}^{C}$, we arrive at the impact parameter space version of the equations of [11]. Now, while these authors solve twodimensional integral equations for the on-shell transition amplitudes, we instead make the additional transformation to the impact parameter representation to eliminate the angular integrations. This difference in calculational procedure being of a technical nature only, it is clear that both methods should yield nearly identical results. Indeed, we exactly reproduce [with the "pole" amplitude (BK) as input] the cross-section values denoted by $\mathrm{BK}_{\mathrm{pr}}$ in Table I of [11]. But at the same time our calculations, when taking the JS amplitude as input, did not reproduce their $\mathrm{JS}_{\mathrm{pr}}$ curves. In fact, the lower the energy was, the larger the discrepancies became. For instance, at $1 \mathrm{keV}$ our cross section for the so-called $\mathrm{JS}_{\mathrm{pr}}$ case is two times bigger than that of [11]. We have checked our results both numerically and analytically, which is easily done because in the $1 s-1 s$ approximation the coupling of the equations becomes rather simple and analytic expressions for all "triangle" graphs in Born approximation are available [14]. Such a large difference can scarcely be blamed on deficiencies of the impact parameter calculation of the total cross section employing straight-line trajectories, for two reasons. First, for the total cross section of light particle excitation or transfer in collisions of two heavy particles at 1 $\mathrm{keV}$, the assumptions implied in an impact parameter calculation are well justified. Second, we exactly reproduce $\mathrm{BK}_{\mathrm{pr}}$ using the same technique. The same group has carried on that work, either by adding more states or by applying their code to other processes. This led, e.g., in [12] and [13], to the unphysical result that at, say $1 \mathrm{keV}$, inclusion of $2 s$ and $2 p$ states increased the total cross section of [11] by $100 \%$, while it is well known that at that energy the process under consideration is completely resonant and the contribution of excited states is negligible. In view of these facts, the lack of agreement between our cross sections and theirs no longer comes as a surprise.

Let us continue the discussion of our results. If the excellent reproduction of the total cross section in CDW and $\mathrm{B} 1 \mathrm{~B}$ were due to a high quality of the transition amplitudes, then this should also show up in angular distributions. In Figs. 6-8, differential $1 s-1 s$ electron-transfer cross sections are presented at a projectile energy of 25,60 , and $125 \mathrm{keV}$, respectively. As noted above, the B1B results pertain to cross sections summed over all final states. It appears that our approach leads, on the whole, to a physically more realistic reaction amplitude, and consequently to a better reproduction of the experimental data, than either $\mathrm{CDW}, \mathrm{B} 1 \mathrm{~B}$, or the impact-parameter coupled-state (MS) model of Ref. [39].

It is also of interest to make a comparison with results of the simple, popular reaction models. This is done in Figs. 9-11, at the same energies as before. As has already been mentioned, the main difference between our theory and the CK model is the absence of the direct channel contribution and the violation of the unitarity constraints in the latter. Whereas this deficiency of CK did not destroy the coincidence in the total exchange cross section beyond, say, 60 $\mathrm{keV}$, it evidently has a strong impact on differential cross sections outside the extreme forward direction. Moreover, comparison with JS clearly demonstrates the importance of using the exact Coulomb $T$ matrix in the "triangle" amplitudes, in addition to the direct channel contribution and to unitarization.

Let us add a few remarks. The first two contributions to the effective potentials (12) taken into account presently contain, beside the simple electron exchange, all the terms involving multiple scattering (of all orders) of the two particles belonging to each of the three pairs as described by the respective Coulomb $T$ matrices-but no terms with consecutive multiple rescatterings between two particles belonging to different pairs (cf. Fig. 2). Nevertheless, rescatterings of the latter type (but only via intermediate formation of a bound state of the electron with either projectile or target ion) are incorporated in the transition amplitudes by means of solving the integral equations (4) (cf. Fig. 1). This latter fact also guarantees that all channels are coupled. If, in our approach, multiple scattering effects between the same two particles are switched off for a moment [i.e., $T_{\nu}^{C} \rightarrow V_{\nu}^{C}, \nu$ $=1,2,3$, in Eq. (12)], the close relationship to standard "firstorder" approaches, i.e., approaches which do not contain so-called double-scattering contributions, becomes apparent. For this reason, only comparison with the most successful "first-order" intermediate- to high-energy approaches is deemed appropriate. To be more precise, the MS approach does contain the important coupling to the direct channel and is taken as an example of close-coupling models as applied successfully to ion-atom collisions at intermediate energies. And CDW, although being based on the first-order approximation to the exact transition amplitude, takes into account multiple scattering of the electron, however only partially (namely in the projectile-electron pair), which is why it fails to reproduce the Thomas peak in the differential electrontransfer cross section at high energies. 
Nevertheless, we did not calculate differential cross sections in the $\mathrm{MeV}$ region. For, as mentioned above, insertion of the first-order terms of our effective potentials in the integral equations implies that a given particle, after being multiple scattered off another particle, first forms a bound state with the third particle before rescattering off the latter becomes possible eventually. This limitation could be relaxed by adding the double-rescattering contribution to the effective potential [i.e., the third term in the expansion (12)], although the latter does not change the total cross section appreciably, except for producing the Thomas peak in differential cross sections. Inclusion of the latter is certainly possible but numerically very involved. The B2B calculations by Belkić [41] are an example where the four doublerescattering terms which are possible in the exchange channel (as many remain in the direct scattering channel) were taken into account, but multiple scattering within each pair was excluded and the three-free-particle Green's function was considered in eikonal approximation only. The results obtained there (within the $1 s-1 s$ model) for differential electron transfer cross sections at 60 and $125 \mathrm{keV}$ show, in our opinion, that in this energy region the contribution from the double-rescattering terms mainly serves to fill the dip which occurs in the $\mathrm{B} 1 \mathrm{~B}(1 s)$ differential cross sections. However, $\mathrm{B} 2 \mathrm{~B}$ is not able to remove the unphysical minimum completely. The latter, together with the fact that the minimum also did not disappear in $\mathrm{B} 1 \mathrm{~B}(\Sigma)$, strengthens our conclusions about the primary importance of the coupling to the direct channels.

In Fig. 12, as an example the differential elastic scattering cross section at $60 \mathrm{keV}$ bombarding energy is presented. The corresponding elastic amplitudes are obtained simultaneously with the transfer amplitudes and, hence, contain information about the other channel through the coupling. The excellent reproduction of the data lends additional support to our claim that the reaction amplitudes as calculated in the three-body approach are of high physical significance. The MS calculation, taken from [42], differs appreciably in the extreme forward direction, while the first Born approximation (FBA) results [43], consisting of the two elasticscattering "triangle", amplitudes, overestimate the cross section for larger angles.

All these figures illustrate in detail the great improvement achieved presently over the traditional approaches, in the energy range considered. We stress once more that in our approach all interparticle interactions are treated on the same footing, and hence none of them needs to be "smuggled in" afterwards by means of a phase factor.

Finally, let us try to assess the quality of the on-shell approximation (9) made in our calculations. Indeed, in the case investigated presently of two heavy protons and one light electron which is to be transferred, off-shell effects are expected to be small, because, as compared to the relatively high collision energies considered, the electron bound state energy $\hat{E}$ is small; hence, the total three-body energy $E$ consists essentially only of the projectile kinetic energy. The probability that the electron, while being transferred, changes the energy of the incident proton is $O(m / M)$, where $m(M)$ is the electron (proton) mass. At the same time, the probability that off-shell effects may result from the virtual excitation of electronic bound states is $O(\hat{E} / E)$. That is, off-shell ef- fects are approximately $O(m / M, \hat{E} / E)$. In the present calculations, e.g., at $1 \mathrm{keV}$, off-shell effects are therefore estimated to be about $1 \%$, quickly becoming $O(m / M)$ at higher energies. This estimate agrees well with the numerical evaluation of off-shell effects performed in [45].

\section{SUMMARY AND CONCLUSIONS}

The main goal of this investigation has been to reestablish the three-body integral equations approach as a valuable and powerful tool for calculating (energetic) atomic collision processes. Its strength derives from one of its most salient and useful features, namely that it allows one, in a natural manner, to systematically include the physically possible and most relevant (re)arrangement channels, and thereby to implement two-body and, at least approximately, three-body unitarity. This objective has been exemplified by means of the scattering of protons of hydrogen atoms. For this purpose a simple (i.e., without initial and final state Coulomb "optical" interaction) version of the three-body AGS formalism, transformed into an effective-two-body theory, was introduced. The appropriate, multichannel, Lippmann-Schwingertype integral equations were considered in on-shell approximation. The resulting two-dimensional integral equations for the physical transition amplitudes were then transformed into the impact parameter representation, leading to a set of coupled algebraic equations. The first two terms in a multiple-scattering-type expansion of the effective potentials occurring therein were taken into account, with the second terms ("triangle" amplitudes) containing the exact off-shell two-particle Coulomb $T$ matrices. Results of our calculations for proton-hydrogen collisions, with only the ground state of hydrogen retained in both the direct and the rearrangement channel ( $1 s-1 s$ model), were presented and compared with those obtained by other methods. The total and differential electron transfer as well as differential elastic scattering cross sections agree very well with experimental data over a wide range of (nonrelativistic) incident energy.

On that basis we conclude that the three-body approach can be applied advantageously to atomic collision problems. Moreover, it has the potential to expose in greatest detail the interesting features of the collision process. Of course, it really does not come as a surprise that proton-hydrogen scattering, a genuine three-body problem, is best described within the framework of the ab initio three-body formalism.

The promising results obtained encourage further development. For instance, generalization to an arbitrary number of involved bound states does not alter the basic equations, although it requires considerable additional numerical effort. Also, generalization to multiply charged projectiles is possible and has, in fact, been done practically in $[14,15]$ (but with only the pole approximation to the effective potentials taken into account). That is, the general strategy will remain unaltered in such further development.

A final point concerns the high-energy (say, beyond 1 $\mathrm{MeV}$ ) behavior of the cross sections, which is not discussed in the present paper. We only mention that their asymptotic behavior is governed by the behavior of the effective potentials, as the on-shell approximation ("unitarized Born approximation") used eventually goes over into the genuine Born approximation. The second-order (double-rescattering) 
terms in the expansion (12) of the effective potentials lead to the well known, correct asymptotic behavior. Though over the years much effort has been devoted to this problem, its practical relevance is arguable because it seems doubtful whether a nonrelativistic approach still makes sense when the nonrelativistic double-rescattering terms start dominating the total cross section.

\section{ACKNOWLEDGMENTS}

This work was supported by the Deutsche Forschungsgemeinschaft (Project No. 436 USB-113-1-0), by the Deutscher Akademischer Austauschdienst (DAAD), by the U.S. DOE (Grant No. DE-FG05-93ER0773), and by the Australian Research Council.
[1] J. R. Oppenheimer, Phys. Rev. 31, 349 (1928).

[2] H. C. Brinkman and H. A. Kramers, Proc. Acad. Sci. (Amsterdam) 33, 973 (1930).

[3] J. D. Jackson and H. Schiff, Phys. Rev. 89, 359 (1953).

[4] I. M. Cheshire, Proc. Phys. Soc. London 84, 89 (1964).

[5] Dž. Belkić, R. Gayet, and A. Salin, Phys. Rep. 56, 279 (1979).

[6] Dž. Belkić, S. Saini, and H. S. Taylor, Z. Phys. D 3, 59 (1986).

[7] Dž. Belkić, S. Saini, and H. S. Taylor, Phys. Rev. A 36, 1601 (1987).

[8] D. P. Dewangan and J. Eichler, Phys. Rep. 247, 59 (1994).

[9] J. C. Y. Chen and A. Chen, in Advances in Atomic and Molecular Physics, edited by D. R. Bates and I. Esterman (Academic Press, New York, 1972), Vol. 8, p. 71.

[10] C. S. Shastry, L. Kumar, and J. Callaway, Phys. Rev. A 1, 1137 (1970).

[11] J. Chaudhuri, A. S. Ghosh, and N. C. Sil, Phys. Rev. A 7, 1544 (1973).

[12] N. C. Sil, J. Chaudhuri, and A. S. Ghosh, Phys. Rev. A 12, 785 (1975).

[13] H. Saha, J. Chaudhuri, and N. C. Sil, Phys. Rev. A 14, 2373 (1976).

[14] G. V. Avakov, A. R. Ashurov, L. D. Blokhintsev, A. M. Mukhamedzhanov, and M. V. Poletayeva, J. Phys. B 23, 2309S (1990).

[15] G. V. Avakov, A. R. Ashurov, L. D. Blokhintsev, A. S. Kadyrov, A. M. Mukhamedzhanov, and M. V. Poletayeva, J. Phys. B 23, 4151 (1990).

[16] G. V. Avakov, L. D. Blokhintsev, A. S. Kadyrov, and A. M. Mukhamedzhanov, J. Phys. B 25, 213 (1992).

[17] E. O. Alt, G. V. Avakov, L. D. Blokhintsev, A. S. Kadyrov, and A. M. Mukhamedzhanov, J. Phys. B 27, 4653 (1994).

[18] S. Alston, Phys. Rev. A 42, 331 (1990).

[19] E. O. Alt, A. S. Kadyrov, A. M. Mukhamedzhanov, and M. Rauh, J. Phys. B 28, 5137 (1995).

[20] E. O. Alt, A. S. Kadyrov, and A. M. Mukhamedzhanov, Phys. Rev. A 54, 4091 (1996).

[21] E. O. Alt, A. S. Kadyrov, and A. M. Mukhamedzhanov, J. Phys. B 30, 3659 (1997).
[22] E. O. Alt, A. S. Kadyrov, and A. M. Mukhamedzhanov, Phys. Rev. A 53, 2438 (1996).

[23] E. O. Alt, P. Grassberger, and W. Sandhas, Nucl. Phys. B 2, 167 (1967).

[24] E. O. Alt, W. Sandhas, and H. Ziegelmann, Phys. Rev. C 17, 1981 (1978).

[25] E. O. Alt and W. Sandhas, Phys. Rev. C 21, 1733 (1980).

[26] J. C. Y. Chen and P. J. Kramer, Phys. Rev. A 5, 1207 (1972).

[27] J. H. Newman, J. D. Cogan, D. L. Ziegler, D. E. Nitz, R. D. Rundel, K. A. Smith, and R. F. Stebbings, Phys. Rev. A 25, 2976 (1982).

[28] W. L. Fite, A. C. Smith, and R. F. Stebbing, Proc. R. Soc. London, Ser. A 268, 527 (1962).

[29] G. W. McClure, Phys. Rev. 148, 47 (1966).

[30] J. E. Bayfield, Phys. Rev. 185, 105 (1969).

[31] A. B. Wittkower, G Ryding, and H. B. Gilbody, Proc. Phys. Soc. London 89, 541 (1966).

[32] P. Hvelpland and A. Andersen, Phys. Scr. 26, 375 (1982).

[33] W. Schwab, G. P. Baptista, E. Justiniano, R. Schuh, H. Vogt, and E. W. Weber, J. Phys. B 20, 2825 (1987).

[34] T. F. Tuan and E. Gerjuoy, Phys. Rev. 117, 756 (1960).

[35] H. Knudsen, H. K. Haugen, and P. Hvelpland, Phys. Rev. A 24, 2287 (1981).

[36] U. Schryber, Helv. Phys. Acta 40, 1023 (1967).

[37] L. H. Toburen, Y. Nakai, and R. A. Langley, Phys. Rev. 171, 114 (1968).

[38] L. M. Welsh, K. H. Berkner, S. N. Kaplan, and R. B. Pyle, Phys. Rev. 158, 85 (1967).

[39] R. Shakeshaft, Phys. Rev. A 18, 307 (1978).

[40] P. J. Martin, D. M. Blankenship, T. J. Kvale, E. Redd, J. L. Peacher, J. T. Park, Phys. Rev. A 23, 3357 (1981).

[41] Dž. Belkić, Phys. Rev. A 43, 4751 (1991).

[42] J. M. Wadehra and R. Shakeshaft, Phys. Rev. A 26, 1771 (1982).

[43] N. F. Mott and H. S. W. Massey, The Theory of Atomic Collisions (Clarendon, Oxford, 1965).

[44] J. T. Park, in Proceedings of XII. ICPEAC, Gatlinburg, 1981, edited by S. Datz (North-Holland, Amsterdam, 1982).

[45] G. V. Avakov, L. D. Blokhintsev, O. Dias, and A. M. Mukhamedzhanov, J. Phys. B 27, 3067 (1994). 